\title{
Cardiovascular diseases: what else should cardiologists know?
}

\begin{abstract}
Cardiovascular diseases remain the main cause of death worldwide. More than 17 million people die annually from cardiovascular diseases (CVD), therefore the World Health Organization (WHO) launched on September 22, 2016 a new initiative to tackle the global threat of CVD, including heart attacks and strokes. Recently a close attention was paid on the retinal microvasculature and it's visualization in patients with CVD. The retina is the only place in whole human organism, where are visible vessels of alive person, reflecting early changes in other organs. Taken into account aforementioned we believe that cardiologists should be aware of valuable diagnostic potential of retinal examination, as it could help to detect early stages of disease comparing with assessment of CVD risk by coronary angiography.
\end{abstract}

Submitted: 27 October 2016; Accepted: 04 November 2016; Published online: 09 November 2016

Keywords: Cardiovascular diseases • Retina - Retinopathy • Biomarker

Cardiovascular diseases remain the main cause of death worldwide. More than 17 million people die annually from cardiovascular diseases (CVD) [1]; therefore, the World Health Organization (WHO) launched on September 22, 2016 a new initiative to tackle the global threat of CVD, including heart attacks and strokes [1].

Recently a close attention was paid on the retinal microvasculature [2] and it's visualization in patients with CVD [3-8]. The retina is the only place in whole human organism, where are visible vessels of alive person, reflecting early changes in other organs. Taken into account aforementioned we believe that cardiologists should be aware of valuable diagnostic potential of retinal examination, as it could help to detect early stages of disease comparing with assessment of CVD risk by coronary angiography.

Currently, wehaveahuge armamentarium to perform a retinal exam (direct ophthalmoscope, indirect ophthalmoscope, slitlamp with fundus lens-ophthalmobiomicroscopy, digital fundus camera).
The existing ophthalmoscopy instruments used in hospitals for detection of CVDs are the following: the direct ophthalmoscope, the indirect ophthalmoscope, slit lamp with fundus lens for ophthalmobiomicroscopy, retinal camera.

The direct ophthalmoscope is a handheld instrument, used to examine the fundus directly. It gives greater magnification $(15 \mathrm{x})$ than the indirect ophthalmoscope and provides an erect, virtual image of the retina. The field of view is only about 5 degree, and stereoscopic vision for the examiner is not possible.

Indirect ophthalmoscope is used for fundus examination because of its larger field, depth of focus, stereopsis, good illumination and produces an inverted and reversed real image on the proximal side of a handheld condensing lens, onto which the examiner accommodate.

Ophthalmobiomicroscopy is the method of examination of the back of the eye, which can be performed on patient's seated at the slit-lamp bio-microscope. As with the
Marianne Shahsuvaryan*

Chinese Academy of Medical Sciences and Peking Union Medical College, Yerevan, Armenia

*Author for correspondence:

Tel.: +37410523468

mar_shah@hotmail.com 
indirect ophthalmoscope, the slit-lamp affords the examiner stereoscopic vision. Handheld plus-diopter condensing lenses can be used, providing the examiner an inverted and reversed retinal image, a field of view ranging between 30 and 40degree, and magnification between $7.5 \mathrm{x}$ and $10 \mathrm{x}$.

Direct ophthalmoscopy, indirect ophthalmoscopy, ophthalmobiomicroscopy give only single image, which is not possible to save in database and thus to evaluate in monitoring process comparing to other one in follow-up visit.

Digital Retina Cameras or Digital Fundus Cameras are used to capture images of the interior surface of the eye. Many of these Digital Retina Cameras boast features like angle variations, color, red-free and angiography imaging, high grade LCD monitors with easy to use features as well as DICOM compatibility and EMR interfacing. These images of the retina, optic disc, macula and posterior pole are digital, which enables quick transfer and detailed image study as well as side-by-side image comparison and longitudinal tracking over time, but the main disadvantage is a high cost. In summary, any type of mentioned examinations is time-taking, requires expensive devices and trained ophthalmic professionals, and in all cases except fundus camera is not repeatable, not transmittable for use in telemedicine. The latest advance in examination of the retina is represented by the innovative D-EYE Portable Eye and Retinal Imaging System- Fundus Smartphone Adapter easily attached to an Apple or Samsung smartphone, creating an ophthalmic camera for vision care screening and evaluation [9]. D-EYE

\section{References:}

1. http://www.who.int

2. McGrory S, Taylor AM, Kirin M, et al. Retinal microvascular network geometry and cognitive abilities in communitydwelling older people: The Lothian Birth Cohort 1936 study. Br. J. Ophthalmol. 2016, 3090 (2016).

3. Tabatabaee A, Asharin MR, Dehghan MH, et al. Retinal vessel abnormalities predict coronary artery diseases. Perfusion. 28, 232-237 (2013).

4. Balta S, Demirkol S, Arslan Z, et al. Retinal vessel abnormalities and coronary artery diseases. Perfusion. 28, 465 (2013).

5. Gopinath B, Chiha J, Plant AJ. Associations between retinal
Portable Eye and Retinal Imaging System is a portable Fundus Camera -digital ophthalmoscope. D-EYE uses the camera and the light source from the Smartphone, illuminating the interior of the eye for examination. Redirecting the light path through the D-EYE lens eliminates corneal glare, a common problem when using a standard ophthalmoscope. Selecting the type of exam (video or multi-shot), type in the patient name and begin the exam. Upon completion, the exam will be stored in the encrypted D-EYE App for later assessment. Retinal images can be emailed or saved to the camera roll but patient identification will be removed by the app. D-EYE offers (for a fee) an optional HIPAA compliant - mobile to cloud - "Image Vault", allowing to remove retinal images from the smartphone and have them stored safely on the cloud (preferred by institutions and hospitals). The price for device is 435 USD. This device does not require any physician. A photo could be taken by technical worker and sent on-line to an ophthalmologist, who is able to conclude on status of retinal microvasculature, sending his conclusion back to the cardiologist, who may necessitate precautions in CVD patients. Thus, modern non-invasive, time-saving, cost-effective retinal exam could be incorporated in the diagnostic protocols of CVD patients.

\section{Conclusion}

In conclusion, interprovider communication including cardiologist, ophthalmologist and communication with patients is vital to optimizing outcomes of patients sufferring from CVD.

microvascular structure and the severity and extent of coronary artery disease. Atherosclerosis. 236, 25-30 (2014).

6. Phan K, Mitchell P, Liew G, et al. Association between Retinal Arteriolar and Venule Calibre with Prevalent Heart Failure: A Cross-Sectional Study. PLoS ONE 10, e0144850 (2015).

7. Wang SB, Mitchell P, Plant AJH, et al. Metabolic syndrome and retinal microvascular calibre in a high cardiovascular disease risk cohort. Br. J. Ophthalmol. 100, 1041-1046 (2016).

8. Seidelmann SB, Claggett B, Bravo PE, et al. Retinal Vessel Calibers in Predicting Long-term Cardiovascular Outcomes: The Atherosclerosis Risk in Communities Study. Circulation. 2, 116 (2016).

9. https://www.d-eyecare.com 